\title{
MESENCHYMAL STEM CELLS ISOLATED FROM THE HUMAN BONE MARROW: CULTIVATION, PHENOTYPIC ANALYSIS AND CHANGES IN PROLIFERATION KINETICS
}

\author{
Tomáš Soukup ${ }^{1}$, Jaroslav Mokrý ${ }^{1}$, Jana Karbanová ${ }^{1}$, Robert Pytlik ${ }^{2}$, Petr Suchomel ${ }^{3}$, Lenka Kučerová ${ }^{4}$
}

Charles University in Prague, Faculty of Medicine in Hradec Králové, Czech Republic: Department of Histology and Embryology ${ }^{1}$; Teaching Hospital, $1^{\text {st }}$ Medical Faculty in Prague, Czech Republic: $1^{\text {st }}$ Department of Medicine ${ }^{2}$, Department of Clinical Genetics ${ }^{4}$; Hospital Liberec, Czech Republic: Department of Neurosurgery ${ }^{3}$

Summary: Mesenchymal Stem Cells (MSCs) are rare elements living in various organs (e.g., bone marrow), able to differentiate into specialized tissues, such as bone, cartilage, tendon, and myocardium. Since the first description of MSCs by Fridenshtein, several investigators have shown that these cells can also differentiate into chondrocytes, adipocytes, and, at least, in rodents into skeletal myoblasts. Later on, more primitive progenitor cells were characterized, able to give rise not only to limb-bud mesoderm, but also to cells of visceral mesoderm. Those cells were named mesodermal progenitor cells (MPCs). The aim of our study was to characterize and compare the biological properties and spontaneous differentiation potential of two different cell types (MSCs and MPCs) isolated from the human vertebral body bone marrow. The results of our experiments proved that the MPCs can be expanded beyond Hayflick's limit and differed from MSCs in morphology, biological and phenotypic characteristics. Because of their high proliferative and differentiation potential, MPCs can become more attractive source of adult stem cells for therapeutic purposes.

Key words: Bone marrow stromal cells; Mesenchymal stem cells; Mesodermal progenitor cells; Long-term culture; Osteogenesis; Chondrogenesis; Adipogenesis; Proliferation kinetics

\section{Introduction}

Stem cells (SCs) represent the first cell types that appear in multicellular organism during ontogenesis. SCs are functional through the entire life-span (they can be isolated from embryo, foetus, children, adults and also from tissues taken from autopsy). Because of this fact, stem cells are endowed with special biological properties (i.e., primitive phenotype, high proliferative capacity, wide differentiation potential and ability for self-renewal). Generally, stem cells represent the cellular elements capable of unlimited proliferation (due to their self-renewal capacity) and of production of several specialized cell types (according to their origin, stem cells are arranged in hierarchy and differ in their potency; they include totipotent, pluripotent and multipotent cells). Production of a resulting phenotype is directed by local microenvironment (niche). During embryonic development, main function of SCs is formation of primordia of individual organs while in adulthood they are responsible for the tissue homeostasis and regeneration. On the basis of latest pieces of knowledge about embryonic, primordial, fetal and adult tissue-specific stem cells, usage of SCs for the therapy of several tissue or organ diseases represents the most perspective therapeutic tool.

Mesenchymal Stem Cells (MSCs) are rare elements living, for example, in the bone marrow stroma ( 2 to 5 cells per million nucleated cells) (12). These cells play crucial role in bone marrow stroma formation and regeneration. MSCs are nowadays intensively studied not only for their haematopoietic support but also for their ability to differentiate in specialized tissues such as bone, cartilage, tendon, myocardium, etc. Extensive proliferation (4) and differentiation into specialized tissues $(13,14)$ are two typical features of MSCs. Differentiation into specialized tissues could be induced either in vitro or in vivo following transplantation.

Since the first description of MSCs by Fridenshtein (6), who demonstrated ability of the bone marrow (BM) to produce colonies of adherent fibroblast-like cells in fetal calf serum (FCS)-containing medium, that differentiate into bone and adipocytes, several investigators have shown that these cells can also differentiate into chondrocytes, adipocytes, and, at least, in rodents into skeletal myoblasts. MSCs can be isolated also from other mesenchymal tissues 
(for example from adipose tissue (17) or dental pulp (8)) and can be purified on the basis of their ability to adhere to plastic or with monoclonal antibodies conjugated to ferromagnetic particles using positive or negative selection. Reyes et al. $(11,15)$ have demonstrated, that using depletion of $\mathrm{CD} 45^{+}$and glycophorin- $\mathrm{A}^{+}$cells from BM mononuclear cells, it is possible to obtain MSCs known to give rise to limb-bud mesoderm, at the single-cell level, that also differentiate into cells of visceral mesoderm and can be expanded extensively by means of clinically applicable methods. Those cells were named mesodermal progenitor cells (MPCs) or multipotent adult progenitor cells (MAPCs). These immunomagnetically separated and in special media cultivated progenitor cells from adult tissues have even wider differentiation potential than researchers expected. Literature suggests that the capability of MAPCs to differentiate into specialized tissues is very close to the potential of embryonic stem cells (ES). Not only this characteristics is great promise for potential stem cell therapy.

The aim of our study was to characterize and compare the biological properties and spontaneous differentiation potential of two different SCs types isolated from the human vertebral body bone marrow: 1) MSCs (in our study we are using this term for SCs cultivated in media containing high levels of FCS) and 2) MPCs (immunomagnetically separated and in low FCS containing media with growth factors cultivated SCs).

\section{Material and Methods}

\section{Bone Marrow}

$\mathrm{BM}$ was obtained from 11 consecutive patients (healthy donors) undergoing posterior spinal instrumentation or vertebroplasty in the lumbar spine following informed consent according to the guidelines from the Liberec Hospital. Before placing the screws or before vertebral augmentation, using transpedicular approach, 2-5 $\mathrm{ml}$ of BM were aspirated.

BM mononuclear cells obtained by optimized FicollPaque density gradient centrifugation were in 8 cases purified on the basis of their ability to adhere to plastic and in 3 cases were depleted of CD $45^{+}$and glycophorin- $\mathrm{A}^{+}$cells by means of micromagnetic beads (Miltenyi Biotec, Sunnyvale, CA).

\section{Culture conditions}

Cells were cultured on untreated plastic (Sarstedt culture flasks or TPP Petri-dishes) at $37^{\circ} \mathrm{C}$ under aerobic conditions $\left(5 \% \mathrm{CO}_{2}\right)$ with FCS-containing alpha-MEM expansion medium ( $1 \%, 2 \%, 10 \%$, and $20 \%$ of FCS). MSCs/MPCs expansion medium consisted of alpha-MEM (Gibco), FCS (PAA), ascorbic acid 2-phosphate (Sigma), dexamethasone (Sigma), L-glutamine (Gibco), penicillin and streptomycin (Gibco). In some cases, we added EGF and PDGF-BB (Peprotech). Once adherent cells were more than $70 \%$ confluent, they were detached with $0.25 \%$ trypsin-EDTA
(Gibco), counted (using haemocytometer or cell viability analyser Vi-Cell XR 2.03 (Beckman Coulter)) and replated at a 1:3 dilution under the same culture conditions.

\section{Differentiation cultures}

To induce differentiation, we used MSCs/MPCs expansion medium with or without FCS supplemented with relevant factors. Cell differentiation is started after reaching of $70 \%$ confluence in culture flask. Adipogenesis is induced in monolayer by decreasing FCS concentration in differentiation media and adding $100 \mathrm{ng}$ insulin (Sigma) per $1 \mathrm{ml}$ of the media. Differentiated adipocytes are visualized using oil red staining. Chondrogenesis is induced in culture by means of micro-mass techniques in basal media with $50 \mathrm{ng} / \mathrm{ml}$ TGF- $\beta 1$ (Stem Cell Technologies) (7,9). Osteogenesis is induced by cultivation in the osteogenic media containing 0.5 $\mathrm{mM}$ of ascorbic acid (Sigma), $10 \mathrm{mM}$ of $\beta$-glycerophosphate (Sigma) and $0.1 \mathrm{mM}$ of dexamethason (Sigma) either in monolayer or in cell pelets $(7,9)$. Osteoblasts are identified using visualization of their products (collagen type I and osteoid) and histochemical detection of alkaline phosphatase using Naphthol AS-MX Phosphate.

\section{Immunofluorescence}

For detection of cytoskeletal proteins, cells were fixed with methanol at $-20{ }^{\circ} \mathrm{C}$ for 2 minutes and permeabilized with $0.1 \%$ Triton X-100 for 10 minutes. For detection of other intracellular molecules, cells were fixed with $4 \%$ paraformaldehyde at $20{ }^{\circ} \mathrm{C}$ for 10 minutes and permeabilized with $0.1 \%$ Triton X-100 for 10 minutes. For identification of cell surface antigens, cells were fixed with $4 \%$ paraformaldehyde at $20{ }^{\circ} \mathrm{C}$ for 10 minutes. Blocking and diluent solution consisted of phosphate-buffer saline (PBS), 1\% BSA, and 1\% serum (Sigma) from the same species as was the primary antibody. Slides were blocked for 30 minutes, incubated sequentially for 30 minutes each with primary antibodies and fluorescein- or phycoerythrin-coupled anti-mouse or antirabbit IgG antibody. Between each step, slides were washed with PBS containing $0.3 \%$ BSA.

\section{Flow Cytometry}

For flow cytometric analysis cells were detached and stained sequentially with immunofluorescent primary antibodies until analysis with a FACSCalibur (Becton Dickinson). DNA analysis was performed by DNA ConKi kit based on propidium iodide staining, according to the instruction of manufacturer. Percentage of positive cells was determined as a percentage of cells with higher fluorescence intensity than the upper $0.5 \%$ of isotype immunoglobulin control. Classification criteria: $<10 \%$ no expression, $10-40 \%$ low expression, $40-70 \%$ moderate expression and $>70 \%$ high expression.

\section{Karyotyping}

Cells (subcultured at a 1:3 dilution, both early passages and after reaching 65 population doublings) were after 24 
hours cultivation subjected to a 4-hour Demecolcemid (Sigma Aldrich) incubation followed by trypsin-EDTA detachment and lysis with hypotonic $\mathrm{KCl}$ and fixation in acid/alcohol. Metaphases were analyzed after GTG banding using software Ikaros v5.0 (MetaSystems).

\section{Results}

\section{Culture of undifferentiated MSCs/MPCs}

Our initial hypothesis presumed that both MSCs and MPCs are present in the vertebral body bone marrow and can be distinguished from haematopoietic progenitors and from each other. MSCs were cultivated without separation step in the basic expansion media containing 20\% FCS. MPCs were first depleted from cells expressing haematopoietic markers (CD45 and GlyA). Following two depletion steps using MACS immunomagnetic beads, mononuclear cells more than $99.5 \%$ CD45-, GlyA- were plated in $25 \mathrm{~cm}^{2}$ untreated plastic surface culture flasks with special expansion media containing $2 \%$ FCS and growth factors (EGF, PDGF). While MSCs grew quite quickly at the beginning and their growth slowed down after $5^{\text {th }}$ passage, MPCs proliferated slowly during the initial expansion but much faster afterwards.

Total population doublings

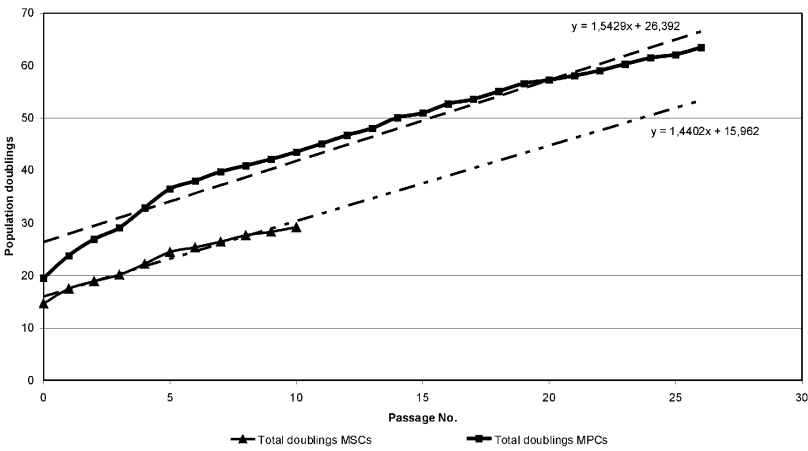

Graph 1: MSCs/MPCs total population doublings - regression, comparison.

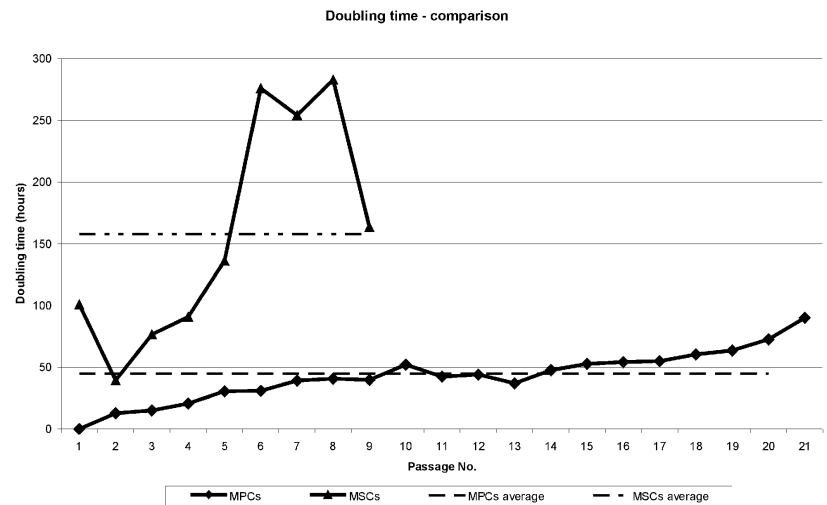

Graph 2: MSCs/MPCs doubling time - comparison.
The growth kinetics of two different bone marrow stromal cells cultures (MSCs, MPCs) was investigated from the primary culture through passage No. 10 in the case of MSCs and through passage No. 26, corresponding to 63.5 population doublings in the case of MPCs. After 7 to 14 days, small clusters of adherent cells developed in both stromal cells cultures. When clusters appeared, all cells were replated in $25 \mathrm{~cm}^{2}$ untreated plastic surface Petri-dish. Once adherent cells were more than $70 \%$ confluent, they were detached, counted and replated at a 1:3 dilution under the same culture conditions. Cell doubling time for MPCs was 12 to 50 hours for the initial 43 cell doublings and increased from 60 to 90 hours after 55 cell doublings. In comparison cell doubling time for MSCs or MPCs cultured in supplemented serum-free medium was initially higher then 50 hours (Graphs 1,2).

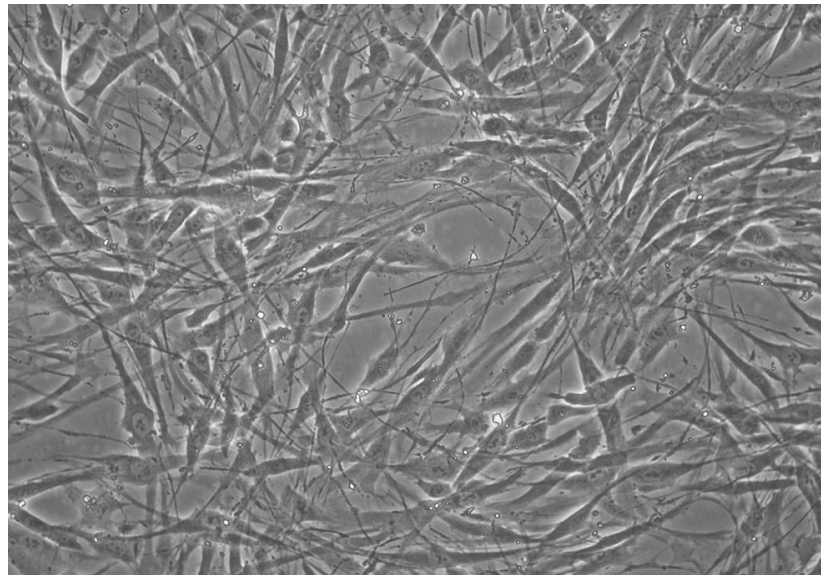

Fig. 1: Typical spindle-shaped morphology of MPCs plated on utreated plastic in expansion medium with $2 \%$ FCS, EGF and PDGF-BB ( 15 cell doublings). MPCs are 10 to 15 $\mu \mathrm{m}$ in diameter.

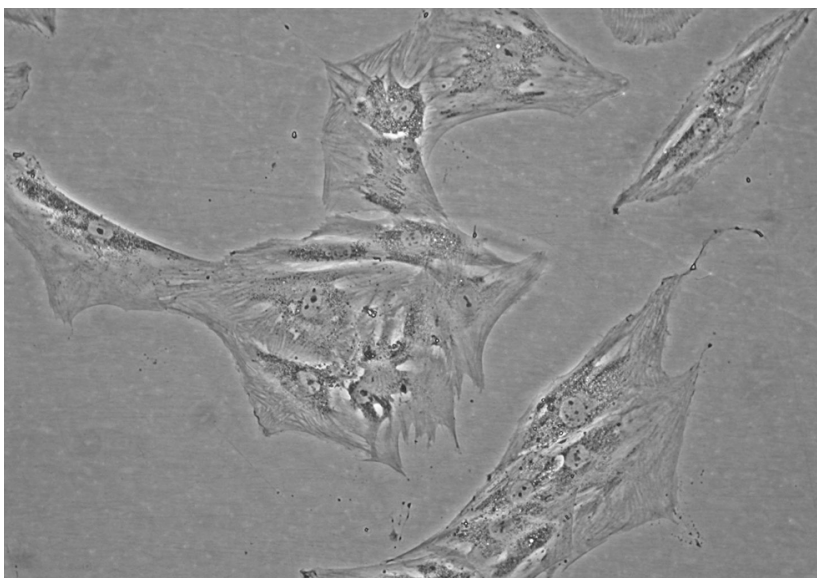

Fig. 2: Typical morphology of MSCs plated on untreated plastic in expansion medium with $20 \%$ FCS without growth factors (10 cell doublings). MSCs assume more flatten morphology and are significantly larger than $20 \mu \mathrm{m}$ in diameter. 

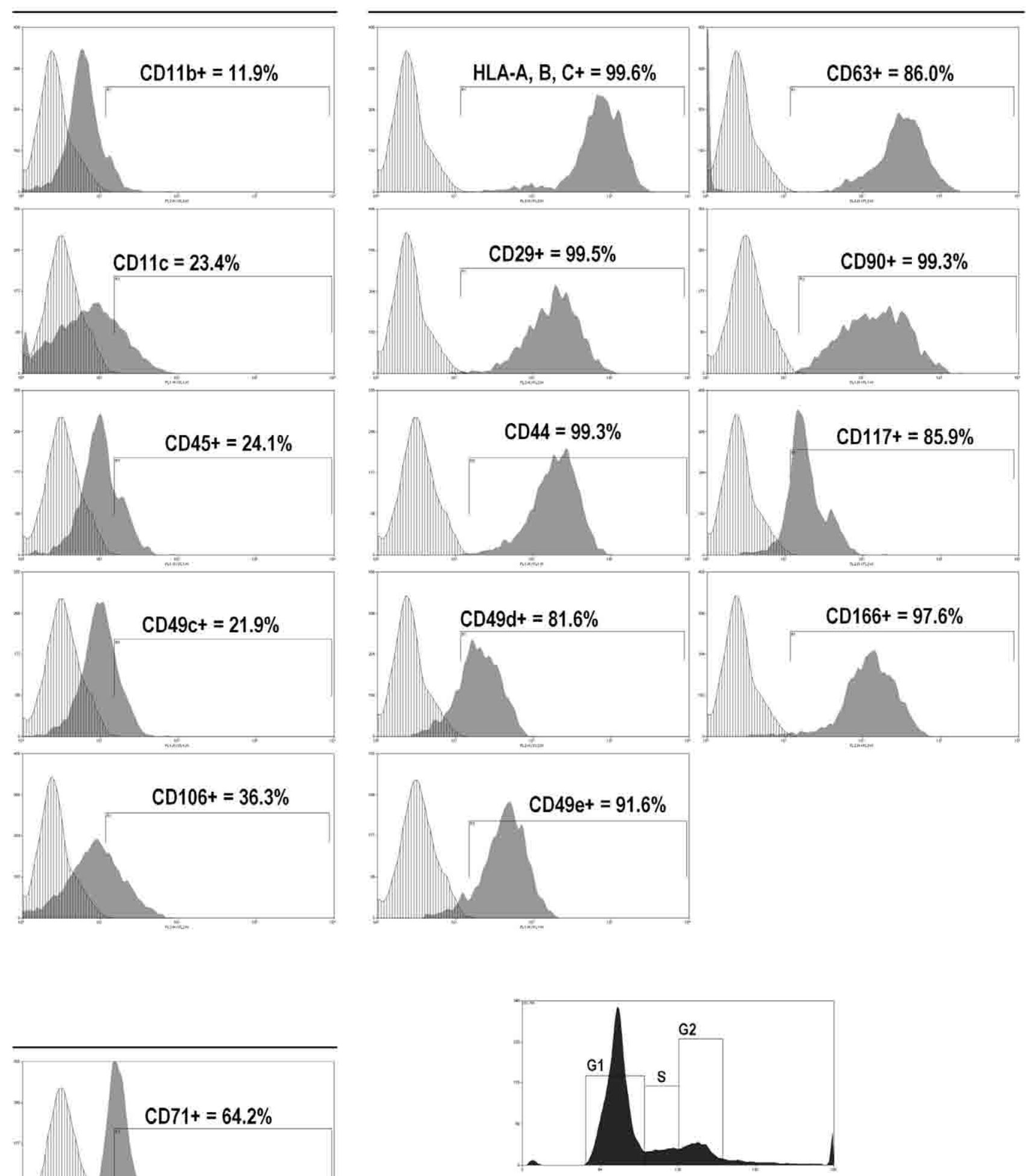

$\mathrm{G} 1=67,2 \% \quad$ If $\mathrm{G} 1+\mathrm{S}+\mathrm{G} 2=100 \%$, then: $S=10,2 \% \quad G 1=73,3 \%$ $\mathrm{G} 2=14,3 \% \quad \mathrm{~S}=11,1 \%$ $\mathrm{G} 1+\mathrm{G} 2+\mathrm{S}=91,7 \% \quad \mathrm{G} 2=15,6 \%$

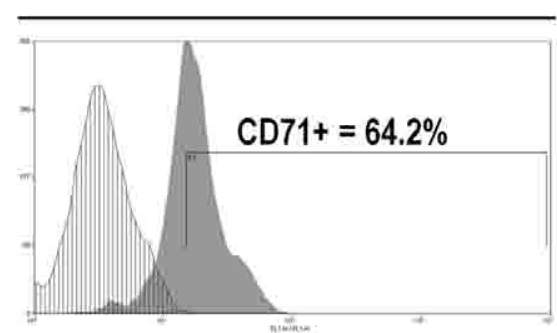
$\mathrm{S}+\mathrm{G} 2=26,7 \%$

Fig. 3: Phenotypic analysis of MPCs plated on untreated plastic in expansion medium with 2\% FCS, EGF and PDGF-BB (10-15 cell doublings). 
It was found that either CD45-GlyA-cells or MSCs, gave rise to clusters of small adherent cells. Those cells differ in amount and morphology according to cell purity, tested plastic type, number of passage and level of FCS in expansion medium (Fig. 1,2). Culture with greater than $2 \%$ FCS or culture at high density yielded cells that were significantly larger (Fig. 2) and later more vacuolated. This was associated with loss of proliferation potential beyond 29 cell doublings.

\section{Cell viability analysis}

During the whole ex vivo expansion process we have also followed the cell viability and other parameters summarized in Tab. 1.

\section{Immunophenotypic analysis}

Immunocytochemical analysis showed that MPCs express low levels of CXCR4 and high levels of telomerase, nestin, fibronectin and STRO-1. Flow-cytometric analysis of cells obtained after 10 to 15 cell doublings showed that cells did not express CD16, CD18, CD34, CD49a, CD68, CD105, CD146, CXCR4, CCR7 and HLA DR, DP, DQ (not shown). Cells expressed low levels of CD11b, CD11c, CD45, CD49c and CD106, medium levels of CD71 and high levels of CD29, CD44, CD49d, CD49e, CD63, CD90, CD117, CD166 and HLA A, B, C. Propidium iodide-based

Tab. 1: Cell viability characteristics of culture-expanded MPCs; representative example of three measurements during ex vivo expansion is shown.

\begin{tabular}{|l|c|c|c|}
\hline MPCs passages & 5 & 15 & 25 \\
\hline Total cells & 792 & 250 & 255 \\
\hline Viable cells & 778 & 245 & 244 \\
\hline Viability (\%) & 98.2 & 98 & 95.7 \\
\hline Total cells / ml (x 10 $)$ & 1.21 & 0.38 & 0.299 \\
\hline Viable cells / ml (x 10 $)$ & 1.19 & 0.377 & 0.289 \\
\hline Average diameter (microns) & 13.3 & 15.5 & 15.5 \\
\hline Average circularity & 0.74 & 0.82 & 0.82 \\
\hline
\end{tabular}

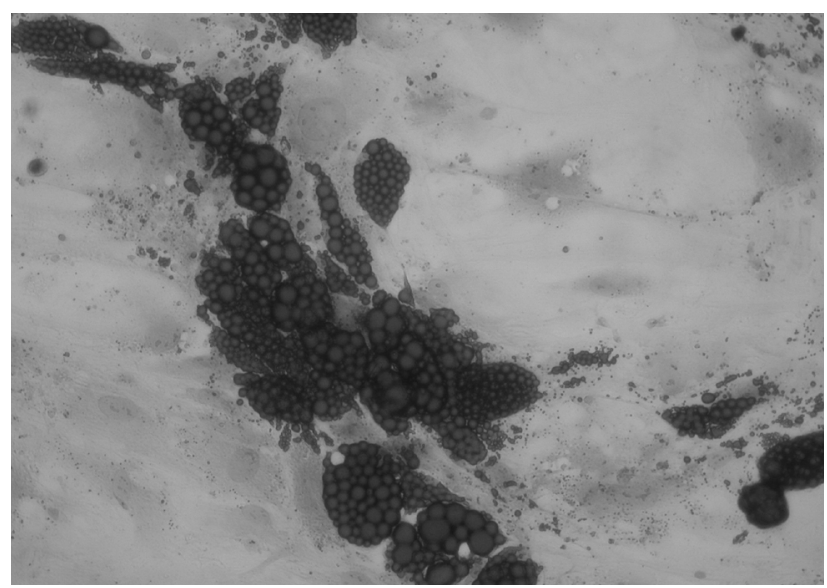

Fig. 4: Adipogenic differentiation from MPCs shown by oil red staining. A representative example of 10 experiments is shown.
DNA analysis showed approximately $25 \%$ of cells being in S-G ${ }_{2}$ phase of cell cycle (Fig. 3).

\section{Adipocytes}

Adipogenesis was induced in MPCs monolayer by decreasing of FCS concentration in differentiation media and adding $100 \mathrm{ng}$ insulin per $1 \mathrm{ml}$ of the media. Cultures were maintained for 14 days, after that, more than $60 \%$ of cells differentiated into lipid-laden cells that stained with oil-red (Fig. 4).

\section{Osteoblasts}

According to the above mentioned protocol we have induced osteogenic differentiation in MPCs monolayer. Cultures were maintained for 4, 8 and 10 weeks. Our results were confirmed by classical histological methods (green trichrome staining, von Kossa), histochemistry (alkaline phosphatase), immunohistochemistry (collagen I) and also transmission electron microscopy.

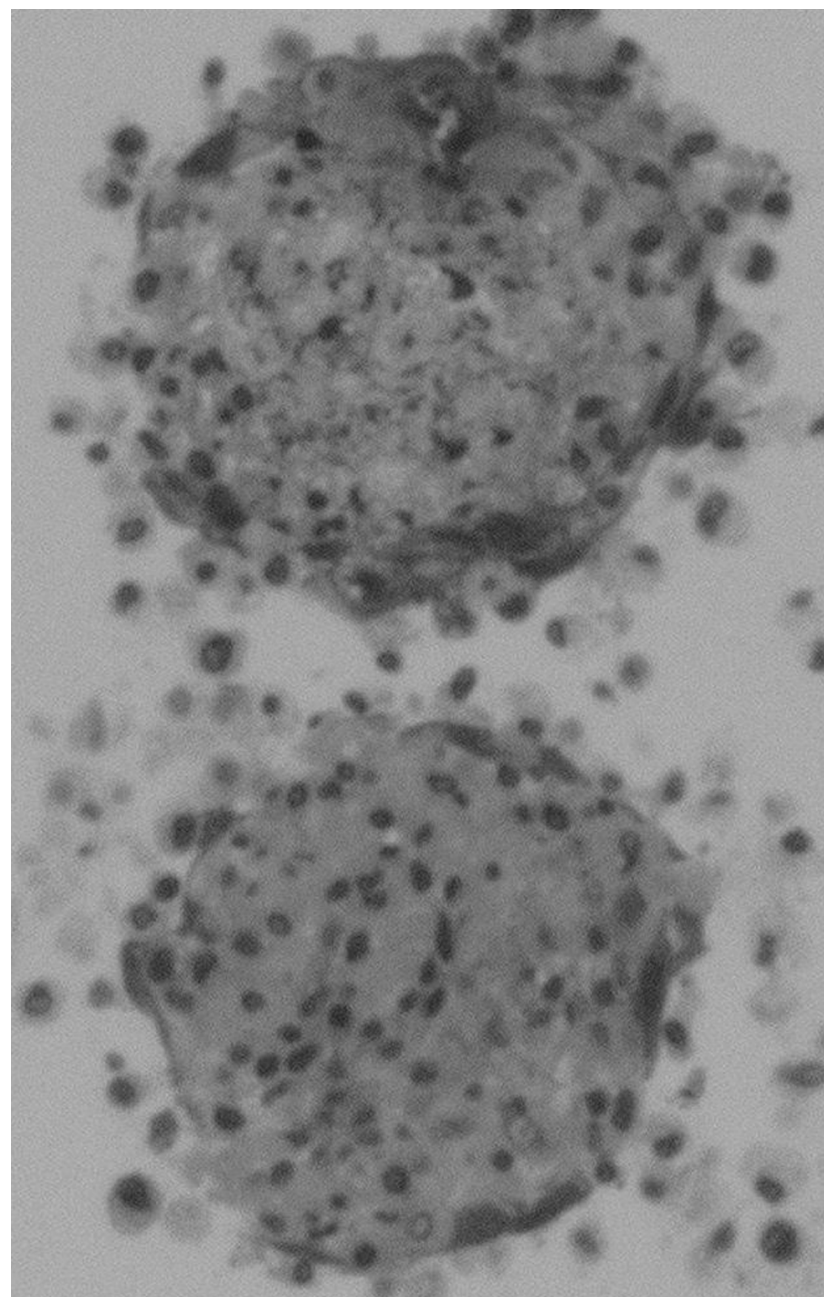

Fig. 5: Chondrogenic differentiation from MPCs using micromass technique is shown by toluidine blue staining. A representative example of 7 experiments is shown. 

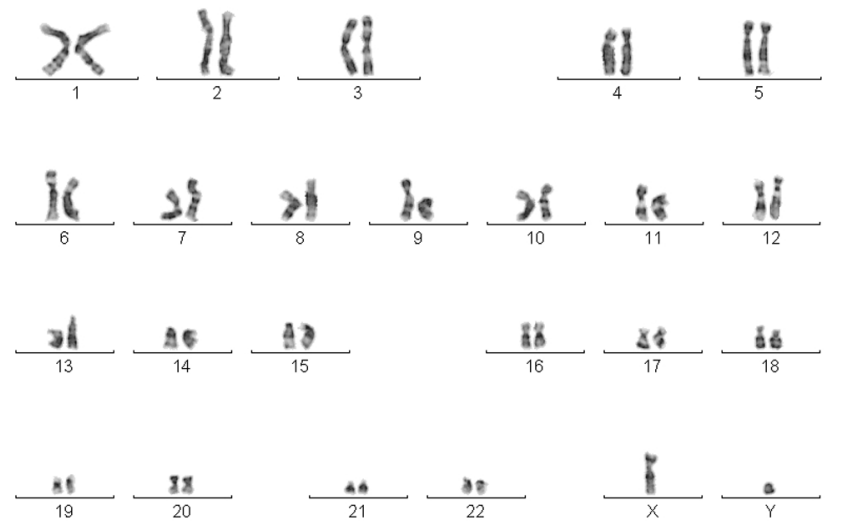

Fig. 6: Karyotyping. CD45-GlyA cells were plated on untreated plastic in expansion medium with $2 \%$ FCS, EGF and PDGF-BB and passaged for 65 cell doublings. Cells were harvested 24 hours after replating and were cytogenetically analyzed. A representative example of 7 experiments is shown.

\section{Chondroblasts}

Development of chondroblasts was followed by staining the micromasses with toluidine blue (Fig. 5), alcian blue and safranin-O and with antibodies against type II and type I collagen.

\section{Karyotyping}

Culture expanded MPCs both primary culture and after reaching of 65 population doublings expressed telomerase and were cytogenetically stable. Cytogenetic examination of cells beyond so called Hayflick's limit (63 population doublings) showed normal karyotype in seven consecutive experiments (Fig. 6).

\section{Discussion}

In the most contemporary stem cell experimental and clinical protocols are the human MSCs prepared with a standard method, with mononuclear cells isolated from an iliac crest bone marrow aspirate using density gradient techniques, and then both enriched and expanded in the presence of high levels of FCS by their tight adherence to plastic tissue culture dishes.

In this report, however, we have shown that relatively homogeneous population of both human MSCs and MPCs can be isolated from a low volume $(2-5 \mathrm{ml})$ of the vertebral body bone marrow. We have described differences in morphological, biological and phenotypic characteristics between MSCs prepared with a standard " $20 \%$ FCS" protocol and MPCs immunomagnetically separated and expanded extensively in vitro in $2 \%$ FCS containing media supplemented with growth factors. As Reyes et al. (15), we were able to cultivate MPCs beyond 50 cell doublings and in contrast with significantly larger and later more vacuolated MSCs, we showed that over the entire cultivation period
MPCs gave rise to clusters of small adherent cells (Fig. 1). Changes in morphology were closely associated with variation of proliferation potential.

Cell doubling time for MPCs was 12 to 50 hours for the initial 43 cell doublings and increased from 60 to 90 hours after 55 cell doublings (Graph 2). This is also corresponding with Reyes et al. (15) observation. Regression analysis of cumulated normal data (population doublings) proved tight dependence of population doublings on passage number. On 5\% level of significance using t-test these two facts were confirmed: 1) Number of population doublings of both MSCs and MPCs did not differ; 2) Doubling time of MSCs cultivated in 20\% FCS is significantly higher than doubling time of MPCs. Moreover, using regression analysis of uncumulated data, we confirmed, that the slowing of cell proliferation is a function of increasing passages $(2,3)$.

MPCs isolated and separated in this study had consistent surface molecule profile as evidenced by 28 available marker antibodies (Fig. 3). While most of these markers have been detected on MSCs as well, some interesting differences can be noted. The levels of CD105 (endoglin) were much lower on MPCs than on MSCs, while levels of CD71 (transferrin receptor), CD117 (c-kit) and CD166 (ALCAM) were higher. CD71 levels on MPCs and MSCs, as on other cell types, correlate with proliferation rate, therefore it is logical, that this marker is more abundant on faster proliferating MPCs. CD166 (ALCAM) correlates positively with osteoblastic potential of MPCs and MSCs (1). While CD117 on MSCs was reported to be negative by most investigators (16), a mesenchymal cell line positive for CD117 with retained potential for osteoblastic differentiation was recently isolated from human hamartoma (5) and CD117 and osteocalcin co-expression was found on activated bone-marrow cells from patients with bone marrow infiltration with haematological malignancies (10). Thus, CD117 expression on MPCs can either indicate their immature status, or their osteoblastic differentiation potential, or their activated status, respectively. In several independent experiments, MSCs expressed only weak staining for CD117.

\section{Conclusions}

We describe here for the first time the isolation and ex vivo culture of mesodermal progenitor cells isolated from the human vertebral body bone marrow. The results of our experiments proved that these MPCs can be expanded beyond Hayflick's limit and differed from MSCs in morphological, biological and phenotypic characteristics. Doubling time of MSCs is significantly higher than doubling time of MPCs. Moreover, we confirmed that the slowing of either MSCs or MPCs proliferation is a function of increasing number of passages. Our MPCs lines expressed consistent surface molecules as evidenced by 28 available marker antibodies. Over the entire cultivation period we did not observe any changes in cell viability and differentiation potential among MSCs and MPCs. Thus, because MPCs can be selected 
and expanded extensively by clinically applicable methods, they may be an ideal source of adult stem cells for the cell therapy.

\section{Acknowledgments:}

The authors wish to thank P. Barsa, M.D., and P. Buchvald, M.D. for help with transpedicular bone marrow harvesting, Ms. Mládková for karyotyping, Ms. Rückerová and $\mathrm{Mr}$. Aoi for help in the tissue cultures laboratory and Mgr. Moos, Ph.D., for introduction into Vi-Cell analysis.

Supported by grant No. 840 23/2005 from Medical Faculty in Hradec Králové and by grant No. ND 7448/3 from IGA MZ.

\section{References}

1. Arai $\mathrm{F}$, Ohneda $\mathrm{O}$, Miyamoto $\mathrm{T}$, et al. Mesenchymal stem cells in perichondrium express activated leukocyte cell adhesion molecule and participate in bone marrow formation. J Exp Med 2002;195(12):1549-1563.

2. Banfi A, Muraglia A, Dozin B, et al. Proliferation kinetics and differentiation potential of ex vivo expanded human bone marrow stromal cells: Implications for their use in cell therapy. Exp Hematol 2000;28(6):707-15.

3. Bruder SP, Jaiswal N, Haynesworth SE. Growth kinetics, self-renewal, and the osteogenic potential of purified human mesenchymal stem cells during extensive subcultivation and following cryopreservation. J Cell Biochem 1997;64(2): 278-94.

4. Colter DC, Class R, DiGirolamo CM, et al. Rapid expansion of recycling stem cells in cultures of plastic-adherent cells from human bone marrow. Proc Nat Acad Sci USA 2000;97:3213-18.

5. Doiguchi Y, Tsukazaki T, Tomonaga T, et al. Establishment of a clonal human mesenchymal cell line that retains multilineage differentiaton capacity from a spinal hamartoma. Cell Tissue Res 2004;317(3):237-246.

6. Fridenshtein A. Stromal bone marrow cells and the hematopoietic microenvironment. Arkh Patol. 1982;44(10):3-11.

7. Gronthos S, Graves S, Ohta S, Simmons P. The STRO-1 + fraction of adult human bone marrow contains the osteogenic precursors. Blood 1994;84:4164-4173.
8. Gronthos S, Mankani M, Brahim J et al. Postnatal human dental pulp stem cells (DPSCs) in vitro and in vivo. Proc Natl Acad Sci USA 2000;97:13625-13630.

9. Haynesworth SE, Barber MA, Caplan IA. Cell surface antigens on human marrow-derived mesenchymal cells are detected by monoclonal antibodies. Bone 1992;13:69-80.

10. Huss R, Moosmann S. The co-expression of CD117 (c-kit) and osteocalcin in activated bone marrow stem cells in different diseases. Br J Haematol. 2002; 118(1):305-12.

11. Jiang Y, Jahagirdar BN, Reinhardt L, et al. Pluripotency of mesenchymal stem cells derived from adult marrow. Nature 2002;418(4):41-49 + Supplementary Information www.nature.com/nature

12. Minguel JJ, Erices A, Conget P. Mesenchymal stem cells. Exp Biol Med 2001; 226:507-20.

13. Pereira RF, Halford KW, O'Hara MD, et al. Cultured adherent cells from marrow can serve as long-lasting precursor cells for bone, cartilage and lung in irradiated mice. Proc Natl Acad Sci USA 1995;92:4857-61.

14. Pittenger MF, Mackay AM, Beck SC, et al. Multilineage potential of adult human mesenchymal stem cells. Science 1999;284:143-7.

15. Reyes M, Lund T, Lenvik T, Aguiar D, Koodie L, Verfaillie CM. Purification and ex vivo expansion of postnatal human marrow mesodermal progenitor cells. Blood 2001:98(9):2615-2625.

16. Tsai MS, Lee JL, Chang YJ, and Hwang SM. Isolation of human multipotent mesenchymal cells from second-trimester amnioti fluid using a novel two-stage culture protocol. Hum Reprod 2004; 19(6):1450-1456.

17. Zuk PA, Zhu M, Ashjian P et al. Human adipose tissue is a source of multipotent stem cells. Mol Biol Cell 2002;13(12):4279-4295.

Submitted September 2005.

Accepted March 2006

Tomáš Soukup, M.D.,

Charles University in Prague, Faculty of Medicine in Hradec Králové, Department of Histology and Embryology, Simkova 870,

50038 Hradec Králové, Czech Republic. e-mail: soukupto@lfhk.cuni.cz 